\title{
A three-IncRNA signature for prognosis prediction of acute myeloid leukemia in patients
}

\author{
FANGCE WANG ${ }^{*}$, XIAOXUE TIAN*, JIE ZHOU, GUANGMING WANG, WENLEI YU, \\ ZHENG LI, ZHUOYI FAN, WENJUN ZHANG and AIBIN LIANG
}

\begin{abstract}
Department of Hematology, Tongji Hospital, Tongji University School of Medicine, Shanghai 200092, P.R. China
\end{abstract}
Received November 7, 2017; Accepted May 24, 2018

DOI: $10.3892 / \mathrm{mmr} .2018 .9139$

\begin{abstract}
Long non-coding RNAs (lncRNAs) are transcripts characterized by $>200$ nucleotides, without validated protein production. Previous studies have demonstrated that certain lncRNAs have a critical role in the initiation and development of acute myeloid leukemia (AML). In the present study, the subtype-specific IncRNAs in AML was identified. Following the exclusion of the subtype-specific lncRNAs, the prognostic value of lncRNAs was investigated and a three-lncRNA expression-based risk score [long intergenic non-protein coding RNA 926, family with sequence similarity 30 member A and LRRC75A antisense RNA 1 (LRRC75A-AS1)] was developed for AML patient prognosis prediction by analyzing the RNA-seq data of AML patients from Therapeutically Available Research to Generate Effective Treatments (TARGET) and The Cancer Genome Atlas (TCGA) projects. In the training set obtained from TARGET, patients were divided into poor and favorable prognosis groups by the median risk score. The prognostic effectiveness of this lncRNA risk score was confirmed in the validation set obtained from TCGA by the same cut-off. Furthermore, the lncRNA risk score was identified as an independent prognostic factor in the multivariate analysis. As further verification of the independent prognostic power of the lncRNA risk score, stratified analysis was performed by a cytogenetics risk group and revealed a consistent result. The prognostic predictive ability of the risk score was compared with the cytogenetics risk group by time-dependent receiver operating characteristic curves analysis. It was revealed that the combination of the lncRNA risk score and cytogenetics risk group provided a
\end{abstract}

Correspondence to: Dr Wenjun Zhang or Dr Aibin Liang, Department of Hematology, Tongji Hospital, Tongji University School of Medicine, 1239 Siping Road, Shanghai 200092, P.R. China

E-mail: zhangwenjun@tongji.edu.cn

E-mail: lab7182@tongji.edu.cn

${ }^{*}$ Contributed equally

Key words: acute myeloid leukemia, long noncoding RNA, prognostic prediction higher prognostic value than a single prognostic factor. The present study also performed co-expression analysis to predict the potential regulatory mechanisms of these lncRNAs in a cis/trans/competing endogenous RNA manner. The results suggested that LRRC75A-AS1 was highly associated with the target genes of transcription factors tumor protein 53 and ETS variant 6. Overall, these results highlighted the use of the three-lncRNA expression-based risk score as a potential molecular biomarker to predict the prognosis in AML patients.

\section{Introduction}

Acute myeloid leukemia (AML) is a highly heterogeneous hematopoietic malignancy with an incidence of approximately $3 / 100,000$ per year (1). Current treatment approaches have resulted in a cure rate of $35 \%$ in younger AML patients and $15 \%$ in older patients $(2,3)$. Based on the NCCN Guidelines for AML, prognosis prediction methods and therapy selected predominantly based on validated cytogenetics which divide the AML patients into favorable, intermediate and poor risk status $(2,4)$. However, some patients eventually relapse despite the lack of adverse risk factors (5). Therefore, optimized biomarkers are necessary in order to refine the prognosis of AML patients.

Long non-coding RNAs (lncRNAs) are defined as non-protein-coding RNA transcripts longer than $200 \mathrm{bp}$. Recently, certain lncRNAs have been reported to exhibit a role in AML. For example, IncRNA CRNDE has been demonstrated to be associated with the classification and total survival of AML patients through regulating proliferation, apoptosis and cell cycle of AML cells (6). Furthermore, Garzon et al (7) derived a lncRNA score composed of 48 lncRNAs for prognostic prediction of cytogenetically normal AML. Notably, the majority of the 48 lncRNAs associated with survival do not associate with known prognostic gene mutations in the study (7). LncRNA prognostic marker independent of known specific subtype will improve survival prediction of AML.

LncRNAs function through regulation of mRNA expression. Previous studies reported that IncRNAs regulate transcription via local (cis) and long distance (trans) mechanisms. Cis-regulation is identified as when the transcription of an lncRNA affects the expression levels of its neighbor genes. Trans-regulation is when that lncRNA can interact with a transcriptional factor (TF) thereby influencing the expression of 
the TF of target genes. In the competing endogenous (ce) RNA hypothesis, IncRNAs compete with the mRNAs containing the same microRNA (miRNA) response elements for binding the miRNAs, thereby influencing the expression of the miRNA target genes (8).

The present study aimed to build an lncRNA risk score to refine AML patient prognostic classification by analyzing AML patients from Therapeutically Available Research to Generate Effective Treatments (TARGET) and The Cancer Genome Atlas (TCGA) project. Furthermore, we predicted the potential target of the prognostic lncRNAs in cis/trans/ceRNA regulation.

\section{Materials and methods}

Acquisition of TCGA and TARGET AML data. The RNA-seq data and corresponding clinical information of AML patients in TARGET and TCGA project were downloaded from Genomic Data Commons Data Portal (portal.gdc.cancer.gov/). Data of acute promyelocytic leukemia in TCGA were filtered out as the therapy and prognosis for acute promyelocytic leukemia patients differs from other subtypes of AML and there were no acute promyelocytic leukemia patients in the TARGET project. Following further removal of data without complete clinical information, a total of 340 TARGET AML samples and 162 TCGA AML samples were investigated in the present study. Based on the GENCODE project long non-coding RNA annotation file (version 26, GRCh38), we obtained the Reads Per Kilobase per Million (RPKM) mapped reads expression data of lncRNAs from level 3 RNA-seq data of TARGET and TCGA.

Identification of subtype-specific lncRNAs. Differentially expressed subtype-specific lncRNAs were calculated using DESeq (version 1.6.1) (9) by comparing the lncRNA expression of a specific class of AML samples with the other AML samples at selection cut-off fold change $>2$ and false discovery rate $<0.05$. The IncRNAs differentially expressed in a certain AML subtype in both TARGET and TCGA projects were considered subtype-specific lncRNAs.

Statistical analysis. After excluding the subtype-specific lncRNAs, the remaining subtype independent lncRNAs were evaluated in the prediction of patient event-free survival (EFS) by univariable cox regression analysis in the TARGET project. The univariable Cox regression analysis was calculated between lncRNA gene expression presented as $\log _{2}(\mathrm{RPKM}+1)$ and patient EFS as days. LncRNAs were considered significantly correlated with patient survival at a threshold of $\mathrm{P}<0.05$. Random survival forests variable hunting (RSFVH) algorithm was carried out to minimize the lncRNAs selected (10). The error rate in the RSFVH model was calculated by 1,000 permutation runs. Then, the selected lncRNAs were subjected to multivariate Cox regression analysis and the risk score was constructed by estimated regression coefficients in the multivariable Cox regression and expression of lncRNAs. The median risk score was selected in the training set as a cut-off dividing the patients into low- and high-risk groups. The Kaplan-Meier method was used to compare the survival difference between the low- and high-risk groups in training and validation sets. Multivariate Cox analysis and Kaplan-Meier survival analysis of cytogenetic stratified AML patients was used to identify the three lncRNAs expression signature as an independent prognostic factor. The sensitivity and specificity of the lncRNA expression signature was evaluated by the area under the receiver operating characteristic (AUROC) of five years EFS. Cox regression, Kaplan-Meier survival analysis and ROC were performed using Statistical software R (version 3.3.3; www.r-project.org/) and survival package, survival ROC package, time ROC package, survminer package based on software $\mathrm{R}(11,12)$.

Co-expression network construction and investigation of cis/trans/ceRNA regulation. We computed the Pearson correlation coefficient between the mRNA and lncRNA expression levels. The mRNA-lncRNA pairs with absolute Pearson correlation coefficient $>0.5$ and $\mathrm{P}<0.001$ in both TCGA and TARGET projects were selected for co-expression network construction.

We identified the genomic distance of the co-expressed IncRNA-mRNA pairs, and the pairs located at the same chromosome within $300 \mathrm{~kb}$ were identified as potentially cis-regulated.

For trans prediction, we focused on the idea that lncRNA may interact with TFs and trans-regulate the expression levels of the target gene of the TF. We used iRegulon plugin within cytoscape software to predict the potential associated TF from the co-expressed gene set of the given lncRNA (13). The target set of TFs with Normalized Enrichment Score (NES) >5 were considered as significantly enriched.

For ceRNA network analysis, we searched the IncRNAmRNA pairs for the same miRNA seed sequence binding site. The miRNA binding sites of lncRNAs were predicted by miRcode (www.mircode.org/) and the miRNA-mRNA relationships were predicted by Targetscan (www.targetscan.org/).

\section{Results}

In the present study, we identified subtype independent prognostic lncRNAs, constructed a lncRNA risk score and explored the potential lncRNA functional mechanisms in cis/trans/ceRNA regulation based on a co-expression network. The framework of this study was presented in Fig. 1.

Identification of subtype dependent lncRNAs. A total number of 15778 lncRNAs were recorded in current human GENCODE release (version 27). A total of 655 of these lncRNAs had stable expression profiling in both the TCGA and TARGET AML datasets ( $R P K M>1$ and read count $>200$ in $>50 \%$ patients). The heatmap of these IncRNAs in the TARGET and TCGA projects are shown in Fig. 2. To work out subtype independent prognostic lncRNAs, we first identified subtype-specific lncRNAs according to the French-American-British (FAB) system, gene mutations and cytogenetic abnormality.

It was revealed that CRNDE, AP002761.1, AC009506.1, LINC01637, AL391832.2 and LINC02081 were highly expressed in the FAB M5 subtype (Fig. 3A and B).

Gene mutations revealed that LINC00982, WT1-AS and LINC0147 were highly expressed in Flt3-itd mutation for AML; HOTAIRM1 and HOXB-AS3 were highly expressed in 


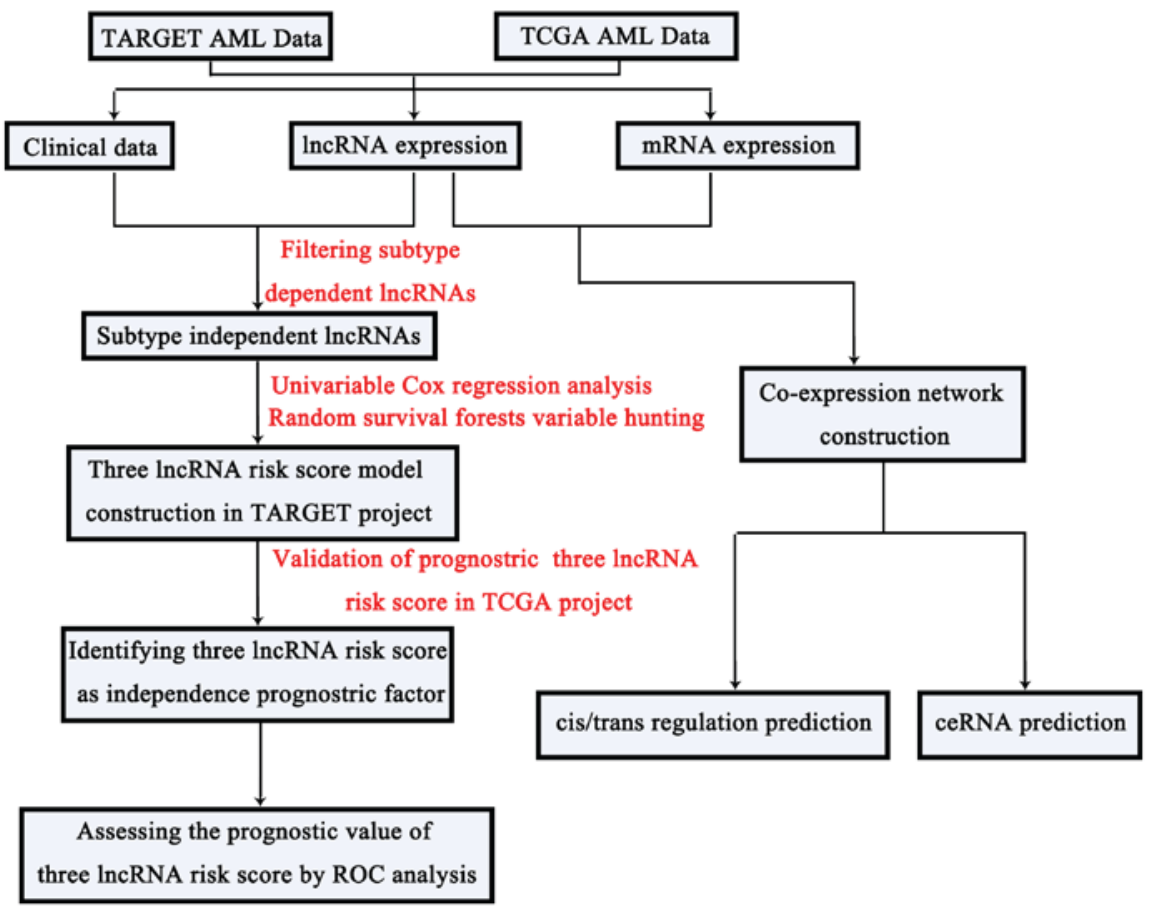

Figure 1. Flow chart of the protocol employed in the present study. TARGET, Therapeutically Available Research to Generate Effective Treatments; AML, acute myeloid leukemia; TCGA, The Cancer Genome Atlas; lncRNA, long non-coding RNA; ceRNA, competing endogenous RNA; ROC, receiver operating characteristic curves.

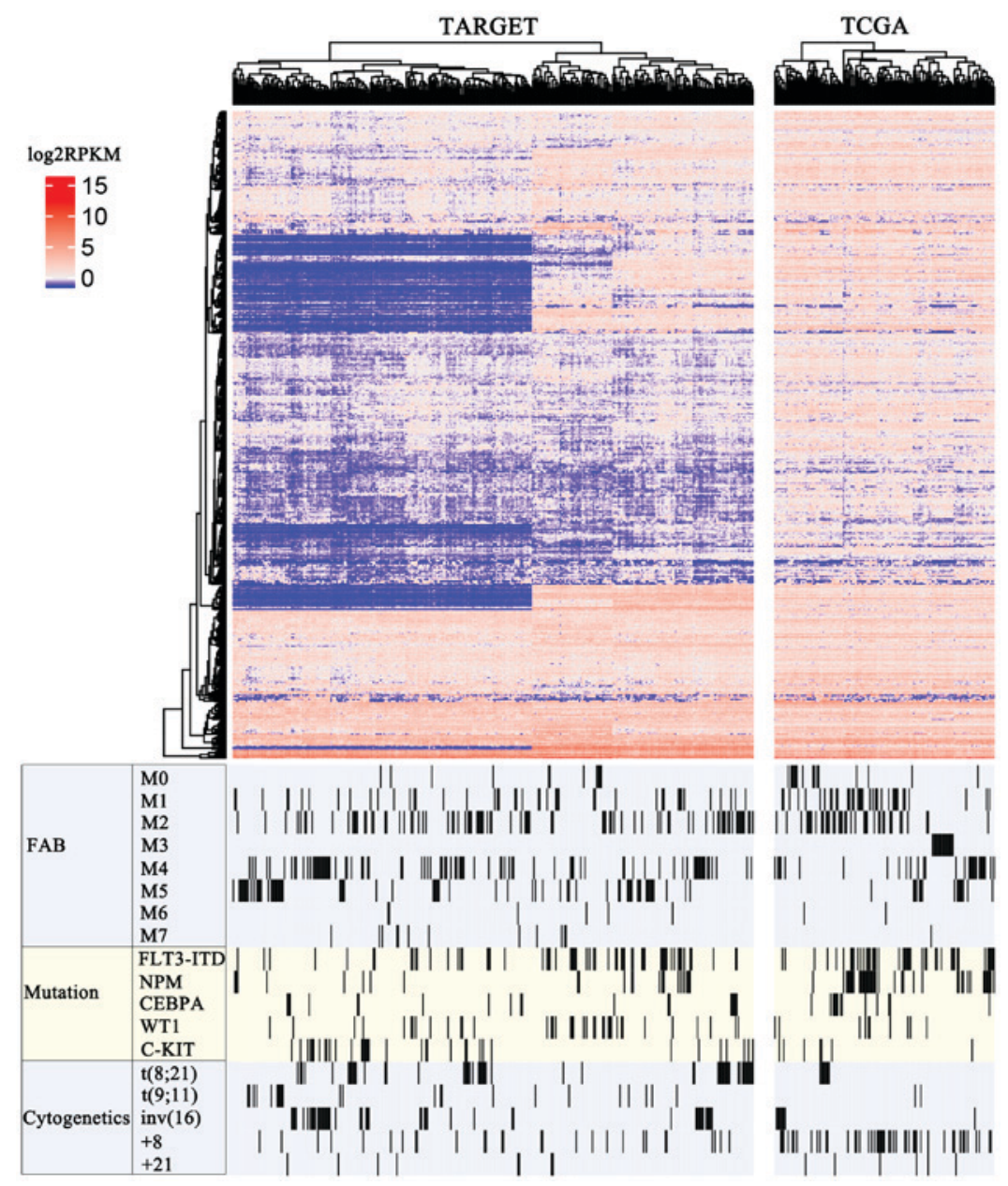

Figure 2. Expression profiles of IncRNAs with stable expression are presented in the heatmap. TARGET, Therapeutically Available Research to Generate Effective Treatments; TCGA, The Cancer Genome Atlas; lncRNA, long non-coding RNA; FAB, French-American-British system; FLT3/ITD, tyrosine kinase 3-internal tandem duplication; NPM, nucleophosmin; CEBPA, CCAAT/enhancer binding protein- $\alpha$; WT1, Wilms tumor 1. 

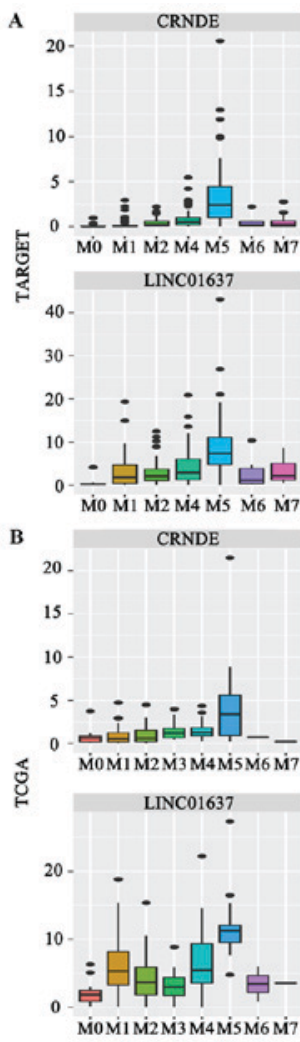
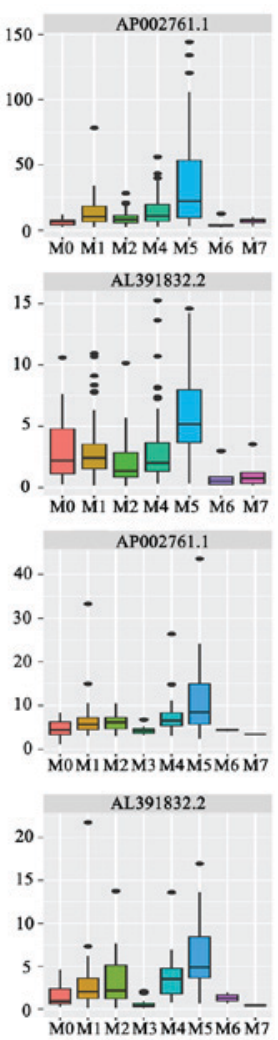
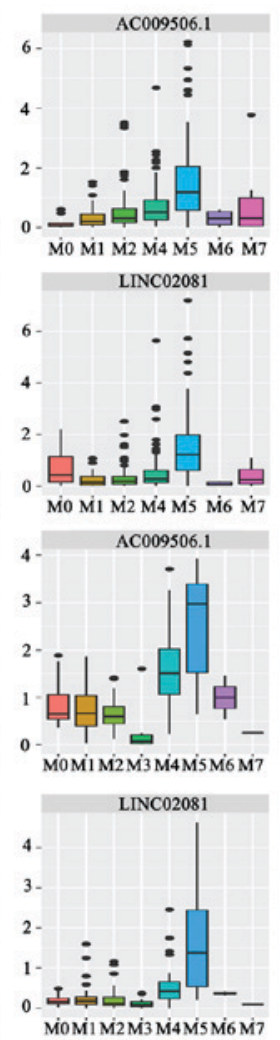

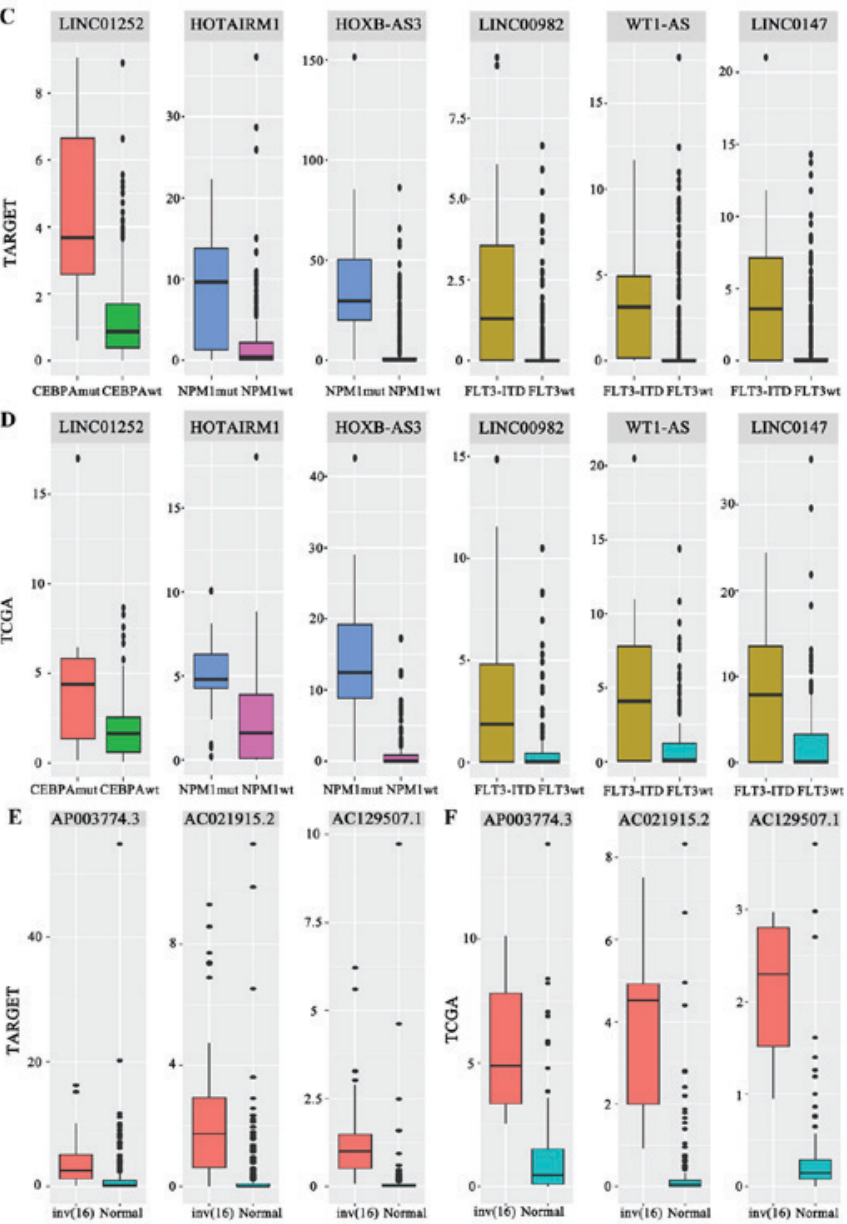

Figure 3. Box plot graphics were employed to illustrate the comparisons in lncRNAs expression between subgroups. (A) Expression of 6 FAB-M5 associated lncRNAs in the TARGET project. (B) Expression of 6 FAB-M5 associated lncRNAs in TCGA project. (C) Expression of 3 FLT3-itd associated lncRNAs, 2 NPM1 mutation associated lncRNAs and a CEBPA mutation associated lncRNA in the TARGET project. (D) Expression of 3 FLT3-itd associated lncRNAs, 2 NPM1 mutation associated lncRNAs and a CEBPA mutation associated lncRNA in TCGA project. (E) Expression of 3 inv (16) associated lncRNAs in the TARGET project. (F) Expression of 3 inv (16) associated lncRNAs in TCGA project. TARGET, Therapeutically Available Research to Generate Effective Treatments; TCGA, The Cancer Genome Atlas; lncRNA, long non-coding RNA; FAB, French-American-British system; wt, wild type; mut, mutation; FLT3-itd, tyrosine kinase 3-internal tandem duplication; NPM, nucleophosmin; CEBPA, CCAAT/enhancer binding protein- $\alpha$; WT1, Wilms tumor 1.

NPM1 mutation for AML; LINC01252 was highly expressed in CEBPA mutation for AML (Fig. 3C and D).

As for cytogenetic abnormalities, we identified that AP003774.3, AC021915.2 and AC129507.1 were highly expressed in AML with inv16 (Fig. 3E and F).

Construction of subtype independent prognostic model based on IncRNA expression. As there were more AML patients in the TARGET database than the TCGA, TARGET AML samples were classified the training set and TCGA AML samples as validation set (14). After filtering out subtype specific lncRNAs, we identified 25 potential prognostic factors that were significantly correlated with the patients' EFS $(\mathrm{P}<0.05)$ in the TARGET project.

To build a more practical model for prediction, we performed the RSFVH algorithm. First, we obtained a prediction with a 0.39 error rate using all 25 potential prognostic lncRNAs as variables (Fig. 4A), and then the variable importance was shown in Fig. 4B.

Then, we calculated the error rate of using smaller number of lncRNAs as variables and found that the error rate was relatively high when using one or two lncRNAs. When using three lncRNA as variables, the lowest error rate combination (LINC00926+LRRC75A-AS1+FAM30A) reached 0.391 which was close to 0.39 (Fig. 4C). Following this, we subjected the three lncRNAs to multivariate Cox regression model for EFS outcome of 340 TARGET AML patients (Table I). The lncRNAs (LINC00926, LRRC75A-AS1) with negative coefficients suggested that higher expressions were associated with favorable survival and the remaining lncRNA (FAM30A) with a positive coefficient suggested higher expression was related to poor survival.

A three-lncRNA signature predicts the survival of AML patients in the training set. We constructed a formula according to the expression level of three lncRNA for EFS outcome in 340 TARGET AML samples using the multivariate Cox regression model as follows: (-0.2763 x expression level of LRRC75A-AS1) + (0.0822 x expression level of FAM30A $)+(-0.1418 x$ expression level of LINC00926). Fig. 5 shows that patients with low-risk scores tended to express high levels of protective lncRNAs (LRRC75A-AS1, LINC00926), whereas patients with high-risk scores tended to express high levels of risky lncRNA (FAM30A). Using the median of risk 
Table I. Multivariable Cox regression of three long non-coding RNAs risk score in Therapeutically Available Research to Generate Effective Treatments acute myeloid leukemia set.

\begin{tabular}{lcccc}
\hline Gene symbol & P-value & Hazard ratio & Variable importance & Relative importance \\
\hline LINC00926 & 0.00699 & 0.8678 & 0.0162 & 0.514 \\
FAM30A & 0.00347 & 1.0749 & 0.0063 & 0.199 \\
LRRC75A-AS1 & 0.00022 & 0.7586 & 0.0316 & 1.000 \\
\hline
\end{tabular}

LINC00926, long intergenic non-protein coding RNA 926; FAM30A, family with sequence similarity 30 member A; LRRC75A-AS1, LRRC75A antisense RNA 1 .
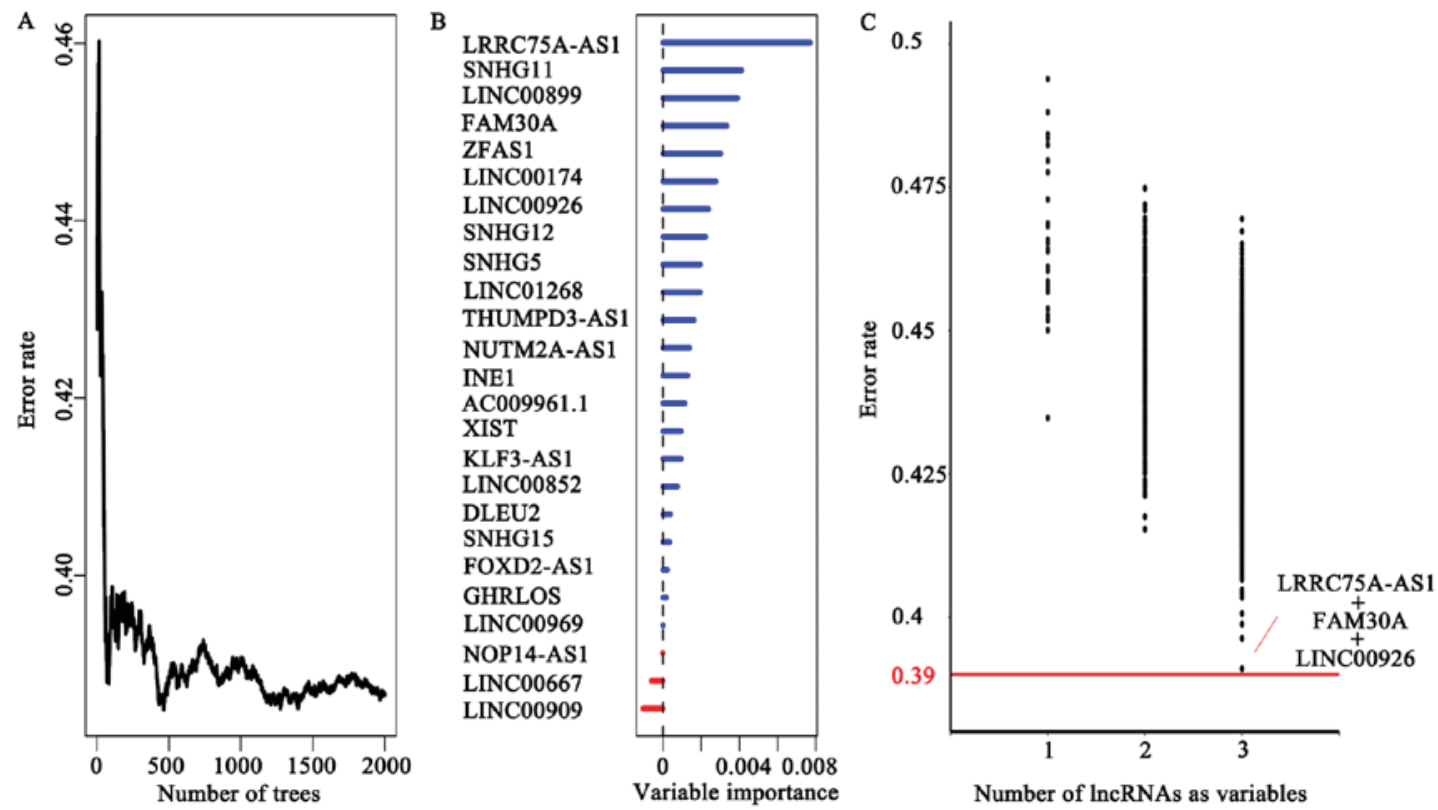

Figure 4. Random survival forests variable hunting analysis for event-free survival in the TARGET database. (A) The error rate of random survival forests model using all 25 potential prognostic lncRNAs as variables. (B) Variable importance values for predictors. (C) The error rate of random survival forests model using 1, 2 and 3 lncRNAs as variables. TARGET, Therapeutically Available Research to Generate Effective Treatments; IncRNA, long non-coding RNA.

score (-2.380) as the cut-off point, patients were divided into a high-risk group (score $>-2.380, \mathrm{~N}=170$ ) and a low-risk group (score $\leq-2.380, \mathrm{~N}=170$ ). We observed that AML patients with high-risk scores had lower EFS rates (log-rank, $\mathrm{P}=0.00012$ ) and OS rates (log-rank, $\mathrm{P}<0.0001)$ compared with those with low-risk scores. To validate our findings, we also classified patients in the TCGA AML set into a high-risk group $(\mathrm{N}=78)$ and a low-risk group ( $\mathrm{N}=84)$ using the same cut-off value. Consistent with the findings described above, patients in the high-risk group suffered significantly poorer EFS (log-rank, $\mathrm{P}=0.0013)$ and $\mathrm{OS}$ (log-rank, $\mathrm{P}=0.011)$ compared with those in the low-risk group (Fig. 6).

A three-lncRNA signature is independent of the cytogenetics risk group. Before evaluating whether the survival prediction based on the three-lncRNA signature was independent of clinical factors, the correlation between clinical factors and the patients' EFS was examined using univariable Cox regression model in TCGA and TARGET. As presented in Table II, we found that only the cytogenetics risk group was closely associated with EFS in all clinical factors. Additionally, multivariate Cox regression analysis revealed that the three-lncRNA signature and cytogenetics risk group were both independent factors in prognosis prediction in the TCGA AML sets, TARGET AML sets and merged sets as presented in Table III.

In the stratified analysis, the merged data set of TCGA and TARGET was divided into favorable, intermediate and poor group according to the cytogenetics risk group and the result showed that the three-lncRNA risk score may further divide AML patients into high-risk and low-risk subgroup within each group (Fig. 7).

Evaluation of the three-lncRNA signature performance by ROC curve analysis. To compare the sensitivity and specificity of the three-IncRNA signature in AML EFS outcome, time dependent ROC curves analysis was performed for the three-lncRNA signature and cytogenetics risk group. The AUROC was determined and compared between these two prognostic factors for five-year EFS in the merged set of TCGA and TARGET. Fig. 8 shows that the AUROC of the three-lncRNA signature was 0.710, and the AUROC of the cytogenetics risk group was 0.692 . There was no significant difference between the AUROC of the three-lncRNA risk score with risk status based on validated cytogenetics and 

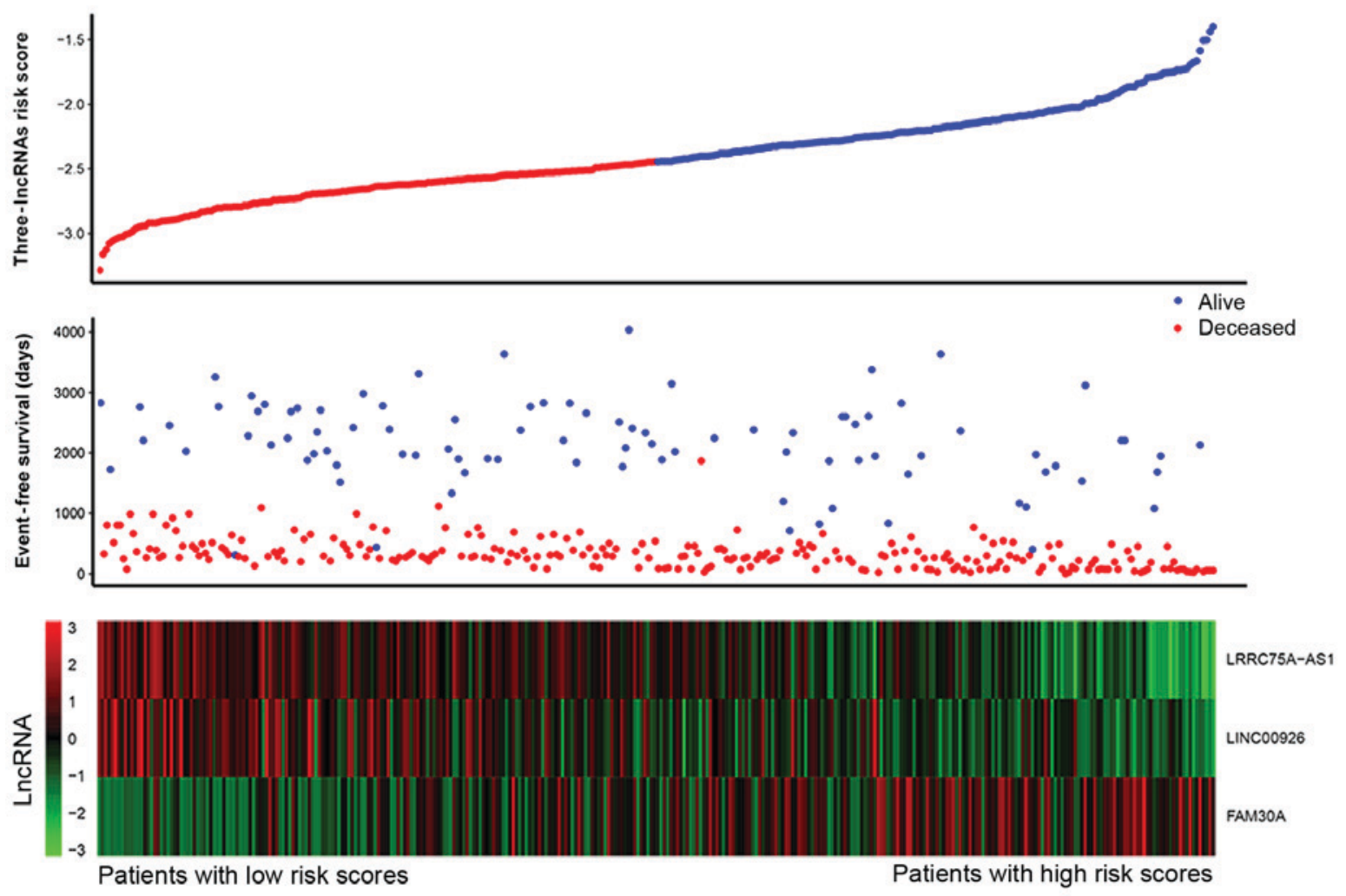

Figure 5. Three-lncRNA based risk score distribution, patients' event-free survival status and a heatmap of the three lncRNA expression profiles. LncRNA, long non-coding RNA.

molecular abnormalities $(\mathrm{P}=0.8574)$. However, we observed that the combination of lncRNA risk score and cytogenetics risk group was a more effective prognostic prediction than each individually (AUROC $=0.764, \mathrm{P}=0.0069$ ).

LncRNAs target prediction and potential regulation mechanisms. At present, the functions of most lncRNAs have not yet been elucidated. To predict the potential target of the three IncRNAs, we constructed a co-expression network based on the Pearson correlation between the mRNAs and lncRNAs of the given lncRNAs in both TARGET and TCGA projects (Fig. 9A). The red nodes in Fig. 8 represent the mRNAs and the blue diamonds indicate the lncRNAs.

In the network, LRRC75A-AS1 regulated most mRNAs (203 mRNAs), followed by FAM30A (99 mRNAs) and then LINC00926 (7 mRNAs). In the result of multivariate Cox regression, these three lncRNAs were independent prognostic factors for AML. No common target was found among the three lncRNAs in this network.

Based on the co-expression network, we explored the potential regulatory mechanism of the IncRNA-mRNA pairs in cis/trans/ceRNA regulation.

A nearby gene that is located $<300 \mathrm{~kb}$ upstream or downstream from the given lncRNA may be the potential cis target. However, none of the three lncRNAs were significantly co-expressed with a nearby gene (data not shown).

As for trans-regulation, it is reported that lncRNA can interact with TFs thereby influencing the expression of the TF target genes. We applied iRegulon to study whether the co-expressed gene sets of the three IncRNAs were overlapping with TF target genes. There were two significant TF enrichments of LRRC75A-AS1 co-expressed genes, TP53 and ETV6. The top scored TF for LRRC75A-AS1 was TP53 with an NES of 7.270 with 38 direct targets, followed by ETV6 (NES=6.473, 52 direct targets) (Fig. 9B). No significant TF enrichments of FAM30A and LINC00926 were identified (Fig. 9B).

In the ceRNA hypothesis, IncRNAs can compete with the mRNAs containing the same miRNA response elements that bind the miRNAs, thereby influencing the expression of the miRNA target genes. We constructed ceRNA networks based on lncRNA/miRNA and miRNA/mRNA interactions. The IncRNA-mRNA pairs that had positively correlated expression profiles and shared at least one common miRNA target were selected for ceRNA network construction. We identified 33 mRNAs as the ceRNA of LRRC75A-AS1, 39 mRNAs as the ceRNA of FAM30A and 6 mRNAs as the ceRNA of LINC00926 (Fig. 9C).

\section{Discussion}

AML is a highly heterogeneous hematopoietic malignancy with a poor outcome. Despite the advances in the risk classification for AML, some patients eventually relapse, even with lack of adverse risk factors. This may result from undiscovered prognostic factors which may refine disease classification. In this study, we first identified the subtype-specific lncRNAs through different expression analysis of clinical and genetic subtypes of AML. Among the subtype-specific lncRNAs, HOTAIRM1 and HOXB-AS3 were reported in previous studies to be upregulated in NPM1-mutated AML, in addition to WT1-AS in AML with Flt3-itd $(7,15)$. 
Table II. Univariable Cox regression of the three-lncRNA risk score and clinical prognostic factors in TCGA and TARGET AML data sets.

\section{A, TCGA set}

\begin{tabular}{lcc}
\hline & & Univariable cox model \\
\cline { 2 - 3 } Variables & HR & $95 \%$ CI of HR \\
\hline Three-lncRNA & 3.689 & $2.230-6.103$ \\
Cytogenetics risk group & 2.179 & $1.629-2.914$ \\
Sex & & \\
Female & 1.002 & $0.6799-1.477$ \\
Male & 0.998 & $0.677-1.471$ \\
Initial WBC & 1.0041 & $0.9997-1.009$ \\
Bone marrow leukemic blast cell percentage & 1.0007 & $0.9946-1.007$ \\
FAB subgroup & & 0.991 \\
AML-M0 & 0.9728 & 0.991 \\
AML-M1 & 1.0244 & 0.068 \\
AML-M2 & 0.9891 & 0.807 \\
AML-M4 & 1.0620 & $0.634-1.655$ \\
AML-M5 & 1.5604 & $0.6171-1.586$ \\
AML-M6 & 2.4879 & $0.6757-1.669$ \\
AML-M7 & 0.7425 & $0.8858-2.749$ \\
Not classified & 2.8980 & $0.6072-10.19$ \\
\hline
\end{tabular}

\section{B, TARGET set}

\begin{tabular}{|c|c|c|c|}
\hline \multirow[b]{2}{*}{ Variables } & \multicolumn{3}{|c|}{ Univariable cox model } \\
\hline & HR & $95 \% \mathrm{CI}$ of $\mathrm{HR}$ & P-value \\
\hline Three-lncRNA & 2.6390 & $1.786-3.900$ & $1.1 \times 10^{-6}$ \\
\hline Cytogenetics risk group & 1.6510 & $1.387-1.965$ & $1.7 \times 10^{-8}$ \\
\hline \multicolumn{4}{|l|}{ Sex } \\
\hline Female & 1.1872 & $0.9247-1.524$ & 0.178 \\
\hline Male & 0.8423 & $0.6561-1.081$ & 0.178 \\
\hline Initial WBC & 1.001 & $0.9997-1.002$ & 0.131 \\
\hline Bone marrow leukemic blast cell percentage & 1.005 & $1.0001-1.0108$ & 0.057 \\
\hline \multicolumn{4}{|l|}{ FAB subgroup } \\
\hline AML-M0 & 1.3073 & $0.48-3.805$ & 0.269 \\
\hline AML-M1 & 0.6137 & $0.4026-1.2354$ & 0.232 \\
\hline AML-M2 & 0.8256 & 0.6201-1.099 & 0.189 \\
\hline AML-M4 & 1.1479 & $0.8618-1.529$ & 0.346 \\
\hline AML-M5 & 1.2127 & $0.8672-1.696$ & 0.260 \\
\hline AML-M6 & 1.3316 & $0.549-3.23$ & 0.526 \\
\hline AML-M7 & 0.7425 & $0.4329-1.274$ & 0.279 \\
\hline NOS & 1.4514 & $0.846-2.49$ & 0.176 \\
\hline Not classified & 1.0976 & 0.6144-1.961 & 0.753 \\
\hline
\end{tabular}

Following exclusion of the subtype-specific lncRNAs, the present study investigated the associations between
lncRNA expression and clinical characters and identified three-lncRNAs (LINC00926, FAM30A, LRRC75A-AS1) 
Table III. Multivariate Cox regression of three-lncRNA risk score and cytogenetics risk status in TCGA, TARGET and merged data sets.

\section{A, TCGA set}

Multivariable cox model

\begin{tabular}{llll}
\cline { 2 - 3 } Variables & HR $\quad 95 \%$ CI of HR P-value
\end{tabular}

\begin{tabular}{llll}
\hline Three-lncRNA & 2.503 & $1.423-4.403$ & 0.0015 \\
Cytogenetics risk group & 1.671 & $1.196-2.336$ & 0.0026 \\
\hline
\end{tabular}

\section{B, TARGET set}

Multivariable cox model

\begin{tabular}{lll}
\cline { 2 - 2 } Variables & HR $\quad 95 \%$ CI of HR & P-value \\
\hline
\end{tabular}

\begin{tabular}{llll}
\hline Three-lncRNA & 2.1371 & $1.438-3.176$ & 0.00017 \\
Cytogenetics risk group & 1.5318 & $1.282-1.830$ & $2.6 \times 10^{-6}$ \\
\hline
\end{tabular}

\section{C, TCGA+TARGET set}

Multivariable cox model

\begin{tabular}{llll}
\cline { 2 - 3 } Variables & HR $\quad 95 \%$ CI of HR & P-value
\end{tabular}

\begin{tabular}{llll}
\hline Three-lncRNA & 2.1591 & $1.539-3.028$ & $8.2 \times 10^{-6}$ \\
Cytogenetics risk group & 1.3519 & $1.153-1.586$ & 0.00021 \\
\hline
\end{tabular}

LncRNA, long non-coding RNA; TCGA, The Cancer Genome Atlas; TARGET, therapeutically available research to generate effective treatments; risk status, risk status based on validated cytogenetics and molecular abnormalities; HR, hazard ratio; CI, confidence interval.

significantly associated with AML patient survival in the training set (TARGET). A prognostic risk score based on the three-lncRNA expression signature was constructed and was efficiently able to divide patients into a different prognostic subgroup. The model was further validated in the testing set (TCGA). It was then confirmed to be an independent prognostic predictor for patients with AML. ROC analysis result showed that the three-lncRNA risk score was competitive for survival prediction. Furthermore, the combination of the three-lncRNA expression signature and the cytogenetics risk group was more informative than each of them individually.

AML patients in TARGET were treated in three clinical trials: CCG2961 (NCT00003790) $(\mathrm{n}=45)$; AAML03P1 (NCT00070174) $(\mathrm{n}=57) ;$ AAML0531 (NCT01407757) $(\mathrm{n}=238)$
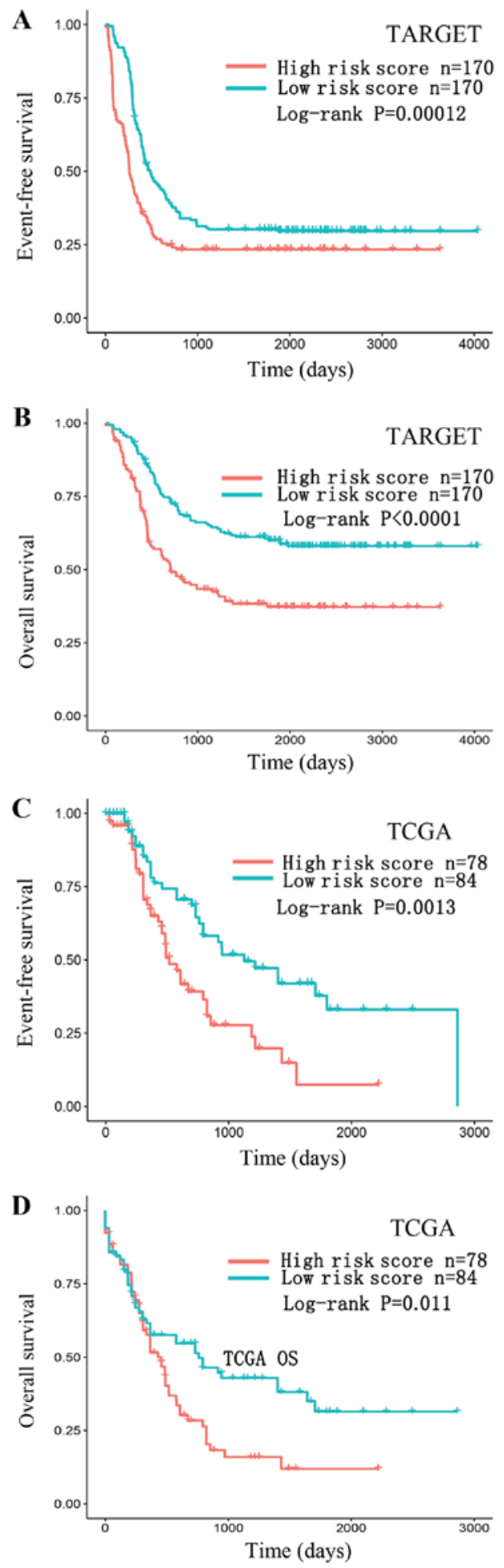

Figure 6. Kaplan-Meier estimates of the survival outcomes for patients using the three-lncRNA signature. (A) Kaplan-Meier curves of event-free survival for the TARGET AML set $(n=340)$. (B) Kaplan-Meier curves of overall survival for the TARGET AML set $(\mathrm{n}=340)$. (C) Kaplan-Meier curves of event-free survival for the TCGA AML set ( $n=162)$. (D) Kaplan-Meier curves of overall survival for the TCGA AML set $(n=162)$. LncRNA, long non-coding RNA; TARGET, Therapeutically Available Research to Generate Effective Treatments; TCGA, The Cancer Genome Atlas; AML, acute myeloid leukemia.

(NCT01723657). Briefly, the induction regimen of CCG2961 was 5-drug combination chemotherapy consisting of dexamethasone, cytarabine, thioguanine, etoposide, and rubidomycin (daunomycin) (DCTER) or IdaDCTER which replaced 

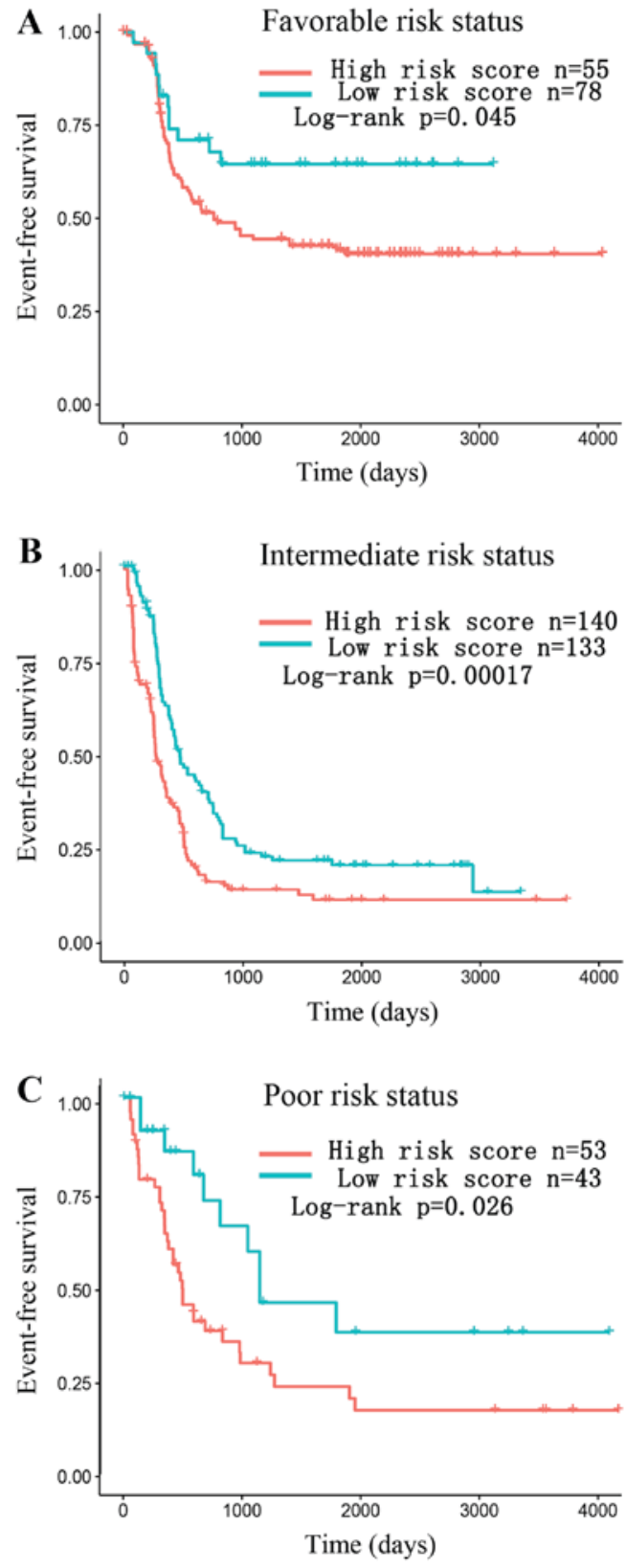

Figure 7. Stratification analyses of all patients adjusted to the cytogenetics risk group. (A) A Kaplan-Meier plot of the favorable risk status patients with AML (n=133). (B) The Kaplan-Meier plot of the intermediate risk status patients with AML ( $\mathrm{n}=273)$. (C) The Kaplan-Meier plot of the poor risk status patients with AML ( $\mathrm{n}=96)$. AML, acute myeloid leukemia.

rubidomycin with IDA at 4:1 ratio in the 5-drug combination while in AAML03P1 and AAML0531, it consisted of standard chemotherapy with or without gemtuzumab ozogamicin. Patients with an unfavorable lncRNA score had lower CR rates in AAML03P1 $(\mathrm{P}=0.0022, \mathrm{OR}=0.0952)$ and AAML0531 $(\mathrm{P}=5.375 \mathrm{e}-11, \mathrm{OR}=0.1422)$. There was no significant different $\mathrm{CR}$ rates in CCG2961 in the comparison between favorable and unfavorable patients $(\mathrm{P}=0.6641, \mathrm{OR}=0.6933)$.

Among the TCGA AML patients, therapeutic regimen consisted of a seven-day continuous infusion of cytarabine plus 3 days of an anthracycline $(7+3)(n=58)$ contained the enough patients for analysis of CR rate and no significant difference in the comparison between favorable and unfavorable patients was observed $(\mathrm{P}=0.6006, \mathrm{OR}=0.7563)$.

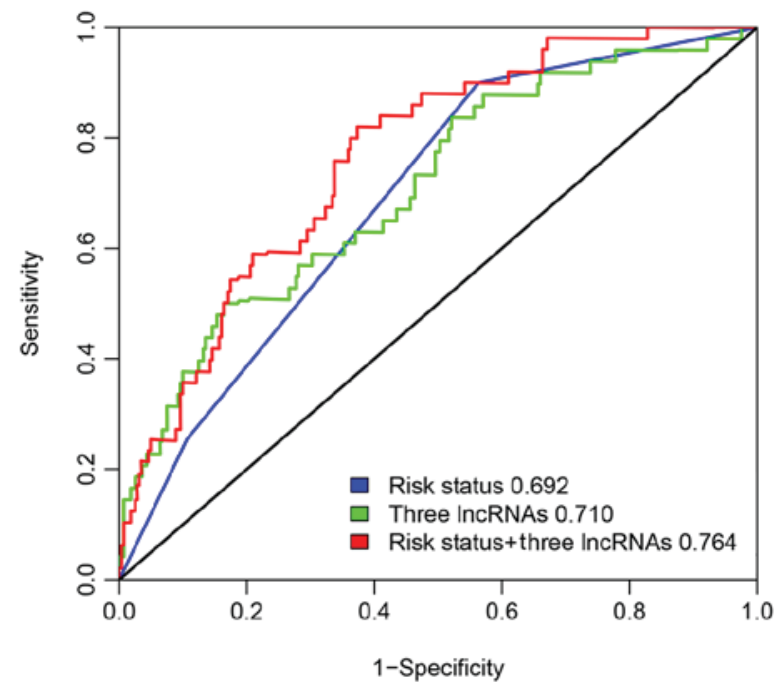

Figure 8. ROC analysis of risk factors for survival prediction in the merged set. The area under the curve was calculated for ROC curves, and sensitivity and specificity were calculated to assess the score performance. ROC, receiver operating characteristic; lncRNA, long non-coding RNA.

It was reported that lncRNAs function through cis, trans and ceRNA regulatory mechanisms to impact the transcription of protein-coding genes $(8,16)$. We identified two significant TF enrichments of LRRC75A-AS1 co-expressed genes, TP53 and ETV6.

TP53 is a well-known tumor suppressor participating in the DNA damage response. TP53-deficient hematopoietic stem cells exhibited high levels of apoptosis and subsequently induction of cancer development under conditions of DNA damage. In a recent study, AML patients with TP53 mutations were defined as an additional AML subgroup and exhibited a worse prognosis (17). The ETV6 gene encodes a transcriptional repressor that plays a critical role in hematopoiesis. Loss of function of ETV6 resulting from mutations or deregulated expression can contribute to leukemogenesis (18).

According to the ceRNA hypothesis, lncRNA acts as a molecular sponge for miRNA thereby regulates the target genes of the miRNA. We constructed the ceRNA network based on the co-expression network. VEGFA was a VEGF family member that was associated with poor prognosis in AML through VEGFA signaling regulating both normal hematopoiesis and AML $(19,20)$. The lncRNA FAM30A may promote VEGFA expression as a ceRNA targeting miR-29-3p.

Previous works have reported some prognostic lncRNAs in AML, such as CRNDE (6), LINC01268 (LOC285758) (21), HOTAIRM1 $(15,22)$, H19 (23). LINC01268 was presented in 25 potential prognostic factors which was significantly correlated with the patients' EFS (Fig. 4). CRNDE and HOTAIRM1 showed a subtype specific expression pattern that they might be more suitable for disease classification than prognosis. H19 was reported as an independent prognostic marker and significantly upregulated in patients with AML-M2. The reason why H19 cannot be validated in TARGET database might be the different between populations and ages. The AML patients in TARGET database was based on pediatric Hispanic or Latino, whereas the AML patients in work of Zhao et al (23) were Chinese adult. 
A

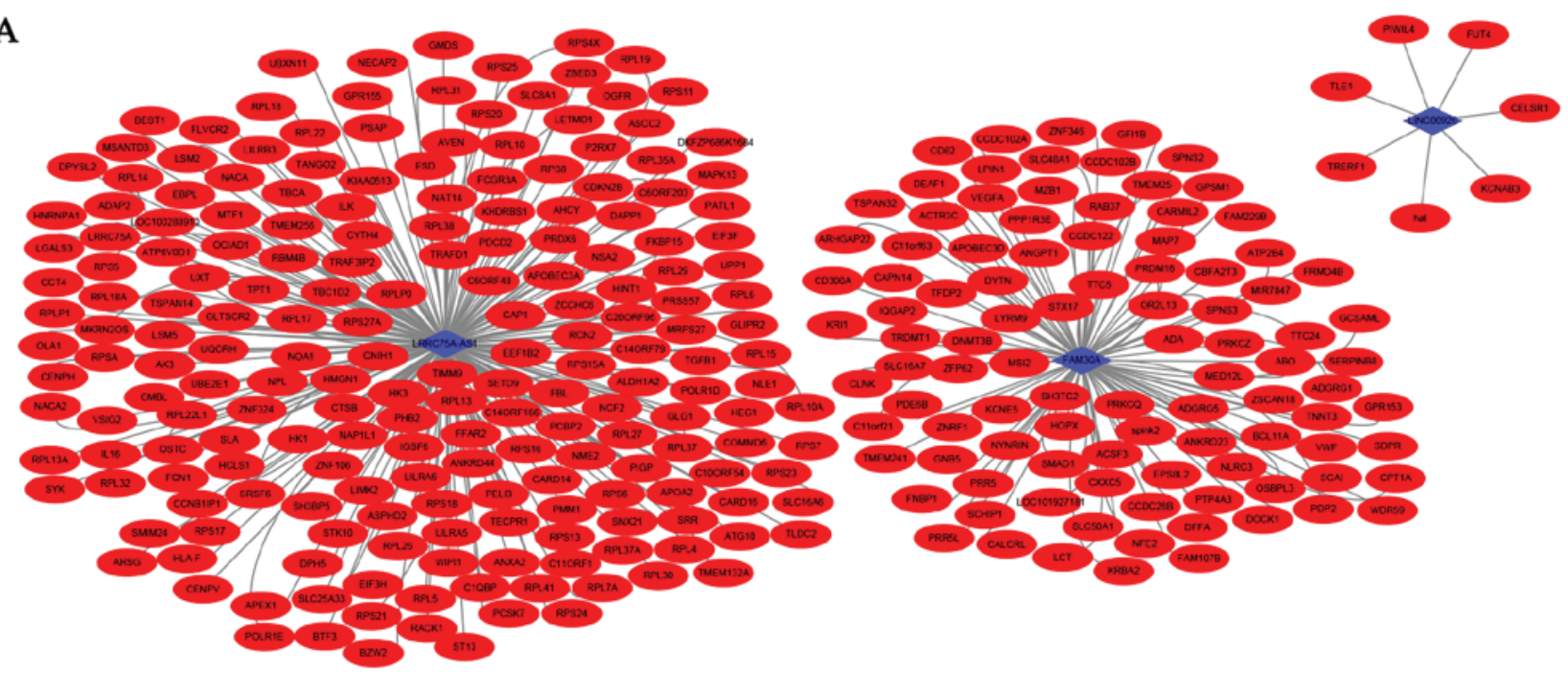

B
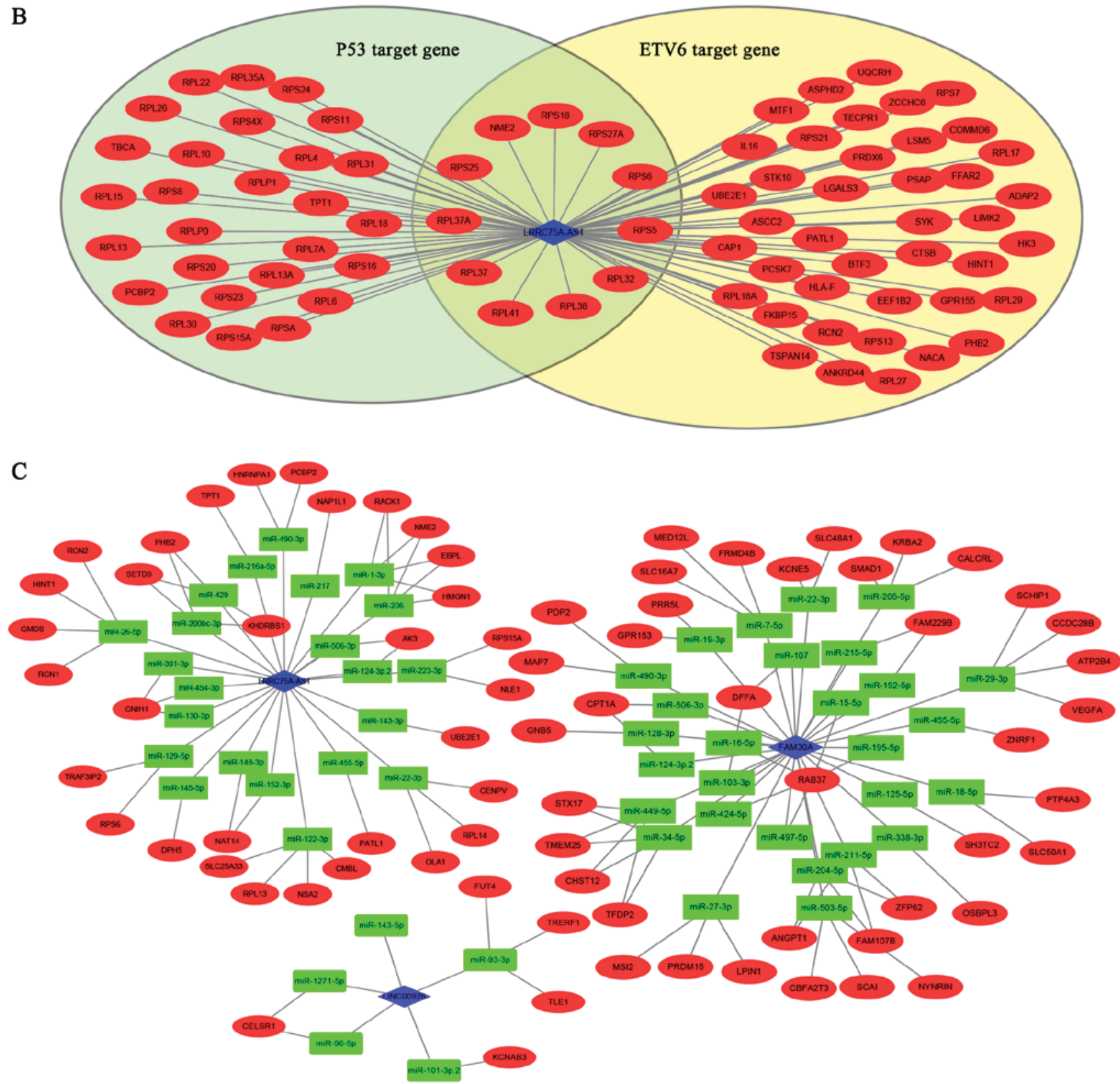

Figure 9. (A) The co-expression network of the three lncRNAs. (B) Network of TP53 and ETV6 target genes associated with LRRC75A-AS1. (C) Competing endogenous RNA network of the three IncRNAs. Red nodes, mRNA; blue diamonds, IncRNA; green rectangle, microRNA. LncRNA, long non-coding RNA; TP53, tumor protein 53; ETV6, ETS variant 6; LRRC75A-AS1, LRRC75A antisense RNA 1. 
Limitation of this study should be acknowledged. First, to make more reliable result for prognosis, we filtered the low expression lncRNAs by RPKM $>1$ and read count $>200$ in $>50 \%$ patients. Thus, various low expression lncRNAs were excluded which might be potentially correlated with AML patients' prognosis. Although TCGA and TARGET projects were both large and comprehensive cancer genomics datasets, the patients were pediatric from TARGET and adult from TCGA. It also revealed that the three-lncRNA risk score was a robust model for survival prediction. Even through the three lncRNAs signature was verified as a prognostic biomarker in TCGA and TARGET project. Experimental studies such as clinical trials were still necessary to avoid the possibilities of false positives. A series of experiments were needed to uncover the lncRNA function and mechanism in cancer.

In conclusion, our data demonstrated that risk score based on three lncRNA expression levels was an independent prognostic biomarker that refined clinical classification. Further multivariate Cox regression analysis has shown that the three-lncRNA signature was an independent prognostic factor. Furthermore, LRRC75A-AS1 may regulate TP53 and ETV6 target genes in trans manner. Potential mRNA-lncRNA pairs were identified as ceRNA based on co-expression network. Practically, the combination of our three-lncRNA signature and cytogenetic risk group achieved a significant improvement in AML patient prognostic prediction compared with either alone.

\section{Acknowledgements}

Not applicable.

\section{Funding}

The present study was supported by the Ministry of Science and Technology of China (grant no. 2016YEE0107200), the National Natural Science Foundation of China (grant no. 81400111) and the Science and Technology Commission of Shanghai Municipality (grant nos. 15411968900 and 15XD1503300).

\section{Availability of data and materials}

The datasets used and/or analyzed during the current study are available from the corresponding author on reasonable request.

\section{Authors' contributions}

WZ and AL conceived and designed the present study. FW and XT performed the statistical analyses and drafted the manuscript. JZ, GW, WY, ZL and ZF performed the experiments and drafted the manuscript. The final version of the manuscript was read and approved by all authors.

\section{Ethics approval and consent to participate}

Not applicable.

\section{Consent for publication}

Not applicable.

\section{Competing interests}

The authors declare that they have no competing interests.

\section{References}

1. Döhner H, Weisdorf DJ and Bloomfield CD: Acute myeloid leukemia. N Engl J Med 373: 1136-1152, 2015.

2. Döhner H, Estey EH, Amadori S, Appelbaum FR, Büchner T, Burnett AK, Dombret H, Fenaux P, Grimwade D, Larson RA, et al: Diagnosis and management of acute myeloid leukemia in adults: Recommendations from an international expert panel, on behalf of the European Leukemia Net. Blood 115: 453-474, 2010

3. Sill H, Olipitz W, Zebisch A, Schulz E and Wolfler A: Therapy-related myeloid neoplasms: Pathobiology and clinical characteristics. Br J Pharmacol 162: 792-805, 2011.

4. Grimwade D, Hills RK, Moorman AV, Walker H, Chatters S, Goldstone AH, Wheatley K, Harrison CJ and Burnett AK: Refinement of cytogenetic classification in acute myeloid leukemia: Determination of prognostic significance of rare recurring chromosomal abnormalities among 5876 younger adult patients treated in the United Kingdom Medical Research Council trials. Blood 116: 354-365, 2010

5. Röllig C, Bornhäuser M, Thiede C, Taube F, Kramer M, Mohr B, Aulitzky W, Bodenstein H, Tischler HJ, Stuhlmann R, et al: Long-term prognosis of acute myeloid leukemia according to the new genetic risk classification of the European LeukemiaNet recommendations: Evaluation of the proposed reporting system. J Clin Oncol 29: 2758-2765, 2011.

6. Wang Y, Zhou Q and Ma JJ: High expression of lnc-CRNDE presents as a biomarker for acute myeloid leukemia and promotes the malignant progression in acute myeloid leukemia cell line U937. Eur Rev Med Pharmacol Sci 22: 763-770, 2018.

7. Garzon R, Volinia S, Papaioannou D, Nicolet D, Kohlschmidt J, Yan PS, Mrózek K, Bucci D, Carroll AJ, Baer MR, et al: Expression and prognostic impact of lncRNAs in acute myeloid leukemia. Proc Natl Acad Sci USA 111: 18679-18684, 2014.

8. Kornienko AE, Guenzl PM, Barlow DP and Pauler FM: Gene regulation by the act of long non-coding RNA transcription. BMC Biol 11: 59, 2013.

9. Anders S and Huber W: Differential expression analysis for sequence count data. Genome Biol 11: R106, 2010.

10. Ishwaran $\mathrm{H}$ and Kogalur UB: Consistency of random survival forests. Stat Probab Lett 80: 1056-1064, 2010.

11. Heagerty PJ, Lumley T and Pepe MS: Time-dependent ROC curves for censored survival data and a diagnostic marker. Biometrics 56: 337-344, 2000.

12. Blanche P, Dartigues JF and Jacqmin-Gadda H: Estimating and comparing time-dependent areas under receiver operating characteristic curves for censored event times with competing risks. Stat Med 32: 5381-5397, 2013.

13. Janky R, Verfaillie A, Imrichova H, Imrichová H, Van de Sande B Standaert L, Christiaens V, Hulselmans G, Herten K, Naval Sanchez M, et al: iRegulon: From a gene list to a gene regulatory network using large motif and track collections. PLoS Comput Biol 10: e1003731, 2014

14. Su Z, Fang H, Hong H, Shi L, Zhang W, Zhang W, Zhang Y, Dong Z, Lancashire LJ, Bessarabova M, et al: An investigation of biomarkers derived from legacy microarray data for their utility in the RNA-seq era. Genome Biol 15: 523, 2014.

15. Díaz-Beyá M, Brunet S, Nomdedéu J, Pratcorona M, Cordeiro A, Gallardo D, Escoda L, Tormo M, Heras I, Ribera JM, et al: The lincRNA HOTAIRM1, located in the HOXA genomic region, is expressed in acute myeloid leukemia, impacts prognosis in patients in the intermediate-risk cytogenetic category, and is associated with a distinctive microRNA signature. Oncotarget 6: 31613-31627, 2015.

16. Cesana M, Cacchiarelli D, Legnini I, Santini T, Sthandier O, Chinappi M, Tramontano A and Bozzoni I: A long noncoding RNA controls muscle differentiation by functioning as a competing endogenous RNA. Cell 147: 358-369, 2011.

17. Taylor J, Xiao W and Abdel-Wahab O: Diagnosis and classification of hematologic malignancies on the basis of genetics. Blood 130: 410-423, 2017.

18. Barjesteh van Waalwijk van Doorn-Khosrovani S, Spensberger D, de Knegt Y, Tang M, Löwenberg B and Delwel R: Somatic heterozygous mutations in ETV6 (TEL) and frequent absence of ETV6 protein in acute myeloid leukemia. Oncogene 24: 4129-4137, 2005. 
19. Santos SC and Dias S: Internal and external autocrine VEGF/KDR loops regulate survival of subsets of acute leukemia through distinct signaling pathways. Blood 103: 3883-3889, 2004.

20. Kim DH, Lee NY, Lee MH, Sohn SK, Do YR and Park JY: Vascular endothelial growth factor (VEGF) gene (VEGFA) polymorphism can predict the prognosis in acute myeloid leukaemia patients. Br J Haematol 140: 71-79, 2008.

21. Lei L, Xia S, Liu D, Li X, Feng J, Zhu Y, Hu J, Xia L, Guo L, Chen F, et al: Genome-wide characterization of 1 ncRNAs in acute myeloid leukemia. Brief Bioinform: Feb 15, 2017 (Epub ahead of print]).

22. Chen ZH, Wang WT, Huang W, Fang K, Sun YM, Liu SR, Luo XQ and Chen YQ: The IncRNA HOTAIRM1 regulates the degradation of PML-RARA oncoprotein and myeloid cell differentiation by enhancing the autophagy pathway. Cell Death Differ 24: 212-224, 2017
23. Zhao TF, Jia HZ, Zhang ZZ, Zhao XS, Zou YF, Zhang W, Wan J and Chen XF: LncRNA H19 regulates ID2 expression through competitive binding to hsa-miR-19a/b in acute myelocytic leukemia. Mol Med Rep 16: 3687-3693, 2017.

(i) () $९$ This work is licensed under a Creative Commons EY NG No Attribution-NonCommercial-NoDerivatives 4.0 International (CC BY-NC-ND 4.0) License. 